\title{
A Novel Technique for Repositioning Lower Eyelid Fat via the Transoral Approach in Association with Midface Lift
}

\author{
M. Mark Mofid
}

Received: 19 August 2010/Accepted: 6 January 2011/Published online: 27 February 2011

(C) The Author(s) 2011. This article is published with open access at Springerlink.com

\begin{abstract}
Background Orbital fat repositioning in association with subperiosteal midface elevation has been variably described via both the transconjunctival and skin muscle flap approaches. Poor visualization, middle and posterior lamellar cicatricial fibrosis, technical difficulty, and incomplete release are disadvantages commonly ascribed to the transconjunctival approach. Lower eyelid malposition and retraction also are commonly seen in association with skin muscle flap approaches. A simple technique using the intraoral approach to release the orbital septum and postseptal fat is described in this report. This procedure avoids complications associated with the violation of key lower eyelid anatomic structures and markedly improves visualization through an incision allowing a much larger access.

Methods A retrospective review of six patients who underwent endoscopically assisted midface elevation in combination with lower eyelid fat repositioning via a transoral approach since 2009 and were followed up for at least 1 year is presented. A pre- and postoperative assessment of tear trough depth, lower eyelid fat herniation, and midface descent is made. Four of the six patients presented also underwent concurrent additional facial rejuvenation procedures including endoscopically assisted brow-lift, genioplasty, structural fat grafting to the nasolabial folds, and lower eyelid "pinch" blepharoplasty. Standard upper buccal sulcus access incisions were used for subperiosteal midface elevation and exposure of the lower orbital
\end{abstract}

M. M. Mofid ( $\square)$

Division of Plastic Surgery, University of California San Diego,

4150 Regents Park Row Suite \#300, La Jolla, San Diego, CA

92037, USA

e-mail:drmofid@mofidplasticsurgery.com septum. Fat redraped over the orbital rim was not secured with fixation sutures as is commonly performed using lower eyelid approaches. Human cadaveric dissection with endoscopic visualization also was performed to demonstrate the reported technique.

Results During this study, one complication developed for a single patient who experienced a temporary dense right zygomatic and frontal branch nerve palsy lasting 8 weeks. No evidence of lower eyelid malposition, ectropion, entropion, incomplete release, or asymmetry was otherwise encountered. Improvement in tear trough appearance, lower eyelid herniation, and midface descent was noted in all six patients.

Conclusion A novel approach for lower eyelid fat transposition in combination with midface lift using the intraoral approach is presented. Excellent visualization, decreased operative times, technical ease, and improved outcomes all are potential advantages of this technique over standard approaches in which access to lower eyelid fat is achieved through the conjunctiva or a skin muscle flap.

Keywords Orbital fat repositioning - Skin muscle flap technique - Subperiosteal midface elevation ·

Transconjunctival technique - Midface lift - Eyelid fat . Lower eyelid fat herniation

\section{Introduction}

Fat transposition techniques for lower eyelid surgery have been advocated for more than a decade [1, 2]. Orbital fat repositioning in association with subperiosteal midface elevation has been variably described via both the transconjunctival and skin muscle flap approaches [3]. Poor 
visualization, middle and posterior lamellar cicatricial fibrosis, technical difficulty, and incomplete release are disadvantages commonly ascribed to the transconjunctival approach [4]. Lower eyelid malpositioning and retraction also are commonly seen in association with skin muscle flap approaches [5].

This report describes a simple technique using an intraoral approach to release the orbital septum and postseptal fat, which avoids complications associated with the violation of key lower eyelid anatomic structures and markedly improves visualization through an incision allowing a much larger access. Given the wide exposure easily achieved via the transoral approach, external and internal fixation sutures commonly applied with other approaches are not used. The additional benefit of combining a midface lift with lower eyelid fat transposition is especially noted for negative-vector and double-convexity patients with a prominent tear trough.

\section{Methods}

\section{Surgical Technique}

The depth of the tear trough deformity is noted preoperatively. Lower eyelid fat herniation and midface ptosis are each assessed and graded in terms of severity as mild, moderate, or severe. Human cadaveric dissection photographs are provided with endoscopic visualization to demonstrate this technique.

Preoperative markings are made on the skin surface overlying lower eyelid fat, the tear trough, and the planned treatment of other facial landmarks such as the nasolabial fold, the trajectory of the frontal branch of the facial nerve, and the zygomatic arch (Fig. 1). The hair is vertically parted, treated with water-soluble lubricating jelly, and secured with small rubber bands or staples within the temporal portion of the hair-bearing scalp. A planned $3-\mathrm{cm}$ incision is marked in this region, with the inferior-most extent approximately $1.5 \mathrm{~cm}$ above the root of the helix of the ear.

A standard wetting solution comprising $50 \mathrm{cc}$ of $1 \%$ lidocaine, $100 \mathrm{cc}$ of injectable normal saline and $0.75 \mathrm{ml}$ of 1:1000 epinephrine is infiltrated bilaterally within the hairline along the planned incision, along the zygomatic arch, in the midface along the path of dissection, and inferiorly within the upper buccal sulcus. A standard upper buccal sulcus incision approximately $3 \mathrm{~cm}$ long then is made using a cutting current on a needle-tipped cautery with care taken to preserve the parotid papilla. At least $5 \mathrm{~mm}$ of gingival cuff is preserved to allow for closure. A molt periosteal elevator is used to elevate the periosteum of the midface, and the extent of the dissection is carried

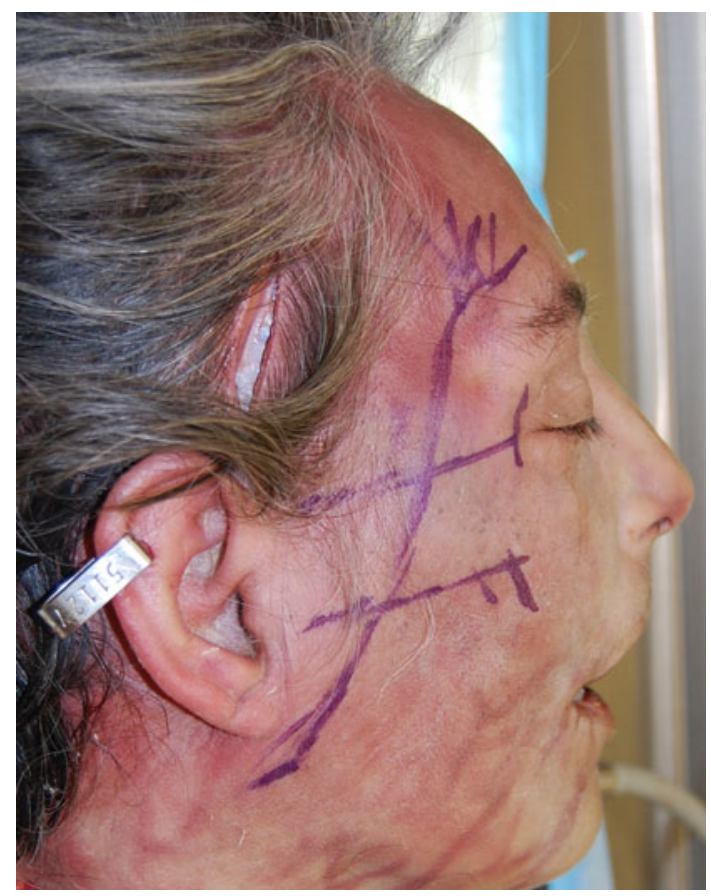

Fig. 1 Preoperative markings demonstrating the trajectory of the frontal branch of the facial nerve, the zygomatic arch and zygoma, and the border of the lateral orbital rim

medially to the nasomaxillary buttress, laterally over the masseteric fascia, superiolaterally over the zygomatic arch, and cranially to the orbital rim dividing the arcus marginalis. Care is taken to preserve the infraorbital nerve at the exit from the infraorbital foramen, and the globe of the eye is protected by an opposite hand placed over the orbital rim during the dissection toward the infraorbital rim.

Once this dissection is complete, an incision is made within the temporal hairline along preoperative markings. Dissection is performed bluntly using a periosteal elevator deep to the superficial layer of the deep temporal fascia in the same plane typically encountered during standard endoscopic or open-brow and midface lift procedures. The dissection may be assisted with an endoscope or through a larger incision via direct visualization depending on the comfort level of the surgeon.

The dissection via the temporal approach is carried inferiorly over the zygomatic arch within a subperiosteal plane, splitting the deep temporal fat pad cranial to the arch. The dissection is continued within the subperiosteal plane over the zygoma inferomedially into communication with the transoral dissection. The medial dissection is carried to the level of the lateral orbital rim. Care is taken to preserve key anatomic structures such as the sentinel vein and the zygomaticofacial nerve within the path of the dissection. A sufficient communication between the temporal and transoral approaches is confirmed by one to two fingers passed over the zygomatic arch (Fig. 2). 


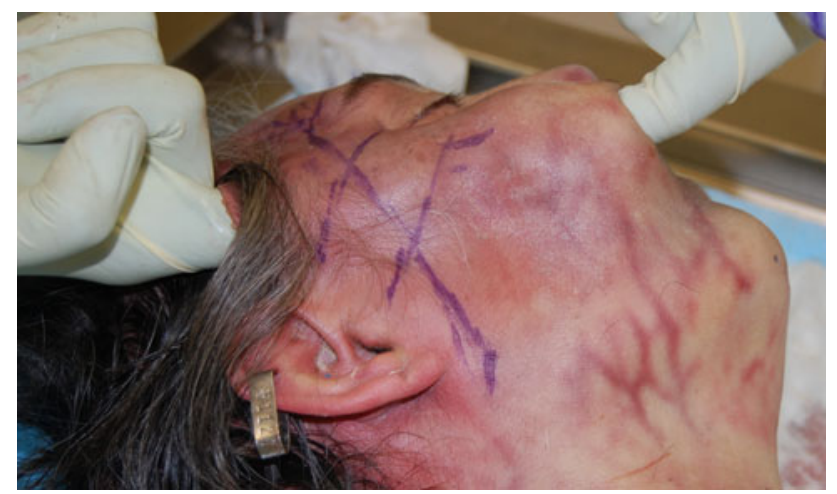

Fig. 2 Confirmation of sufficient communication between the temporal and intraoral dissections by passing fingers between the two planes of dissection

With the dissection and exposure completed, open visualization via the intraoral approach is used to divide the orbital septum. Gentle pressure over the globe with a finger is used to evaluate the amount of bulging and herniation of postseptal fat (Fig. 3). Gorney or long-handled Stevens scissors are used to divide the septum just above the arcus marginalis transversely along the entire length of the orbital rim. Lateral, central, and medial fat pockets are easily teased from their respective compartments using blunt forceps for transposition over the orbital rim (Fig. 4). No fixation is necessary with adequate release.

Depending on the surgeon's preference, fixation devices or sutures may be used to accomplish the midface lift at this point with sutures to be secured to the deep temporal fascia in a path over the zygomatic arch. In the presented cases, the author used the Coapt Endotine Midface ST device (Coapt Systems, Inc., Palo Alto, CA) and secured

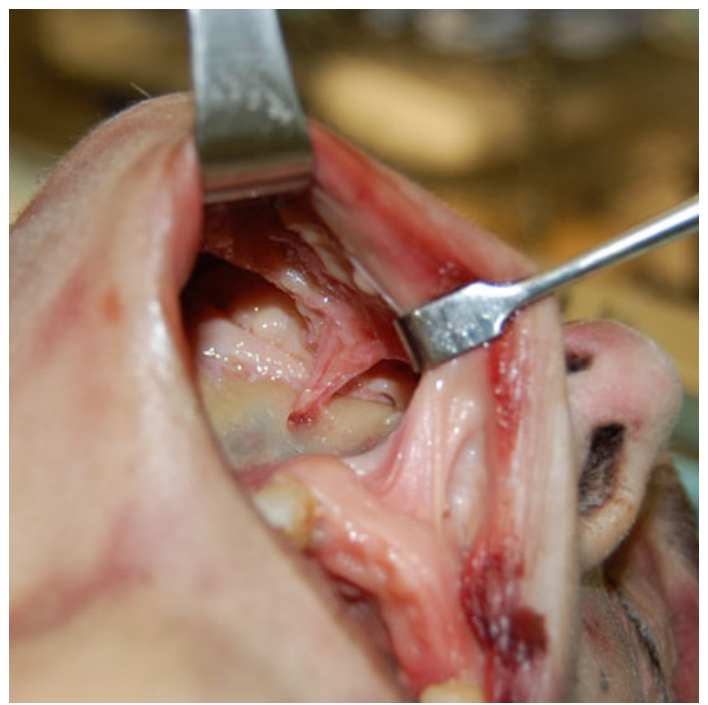

Fig. 3 Postseptal fat is easily visualized through the intraoral dissection

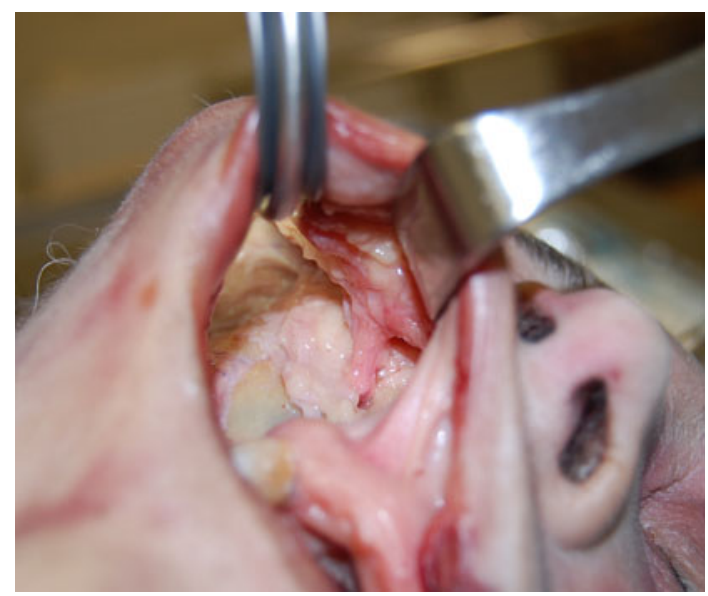

Fig. 4 Postseptal fat is easily teased over the infraorbital rim to allow for stable transposition

the device holes to the deep temporal fascia using 2-0 PDS suture (Fig. 5). The temporal incision then was closed with staples, and the intraoral incision was closed with 3-0 chromic gut suture on a tapered needle.

Patients are instructed to adopt a liquid diet for $48 \mathrm{~h}$ followed by a soft diet for an additional 7 days. Staples are removed at 7 days. Principles outlined by the Department of Health and Human Services Regulations for the Protection of Human Subjects were followed in the performance of this study.

\section{Results}

The study enrolled six consecutive patients who had undergone lower eyelid fat repositioning using the intraoral

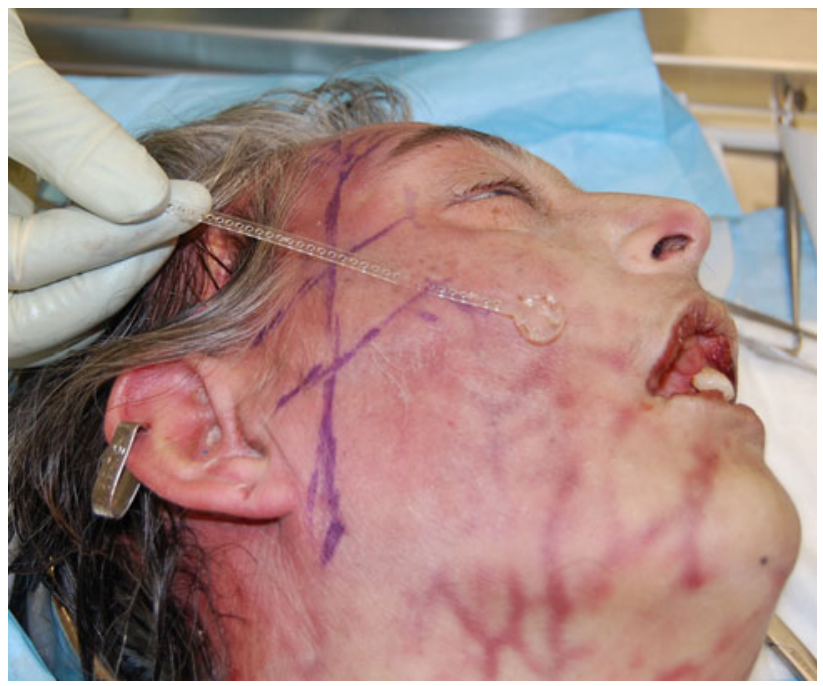

Fig. 5 The Coapt Endotine Midface ST device 
approach in association with midface lift since 2009 (Fig. $6 \mathrm{a}-\mathrm{c}$ ). The minimal follow-up period for the patients included in the study was 12 months. Four of the six patients also had undergone concurrent additional facial rejuvenation procedures including lower eyelid skin-only "pinch blepharoplasty" (one patient) endoscopically assisted brow-lift (three patients), genioplasty (one patient), and structural fat grafting to the nasolabial folds (one patient). Once the dissection and exposure for a midface lift was performed, the average additional time for
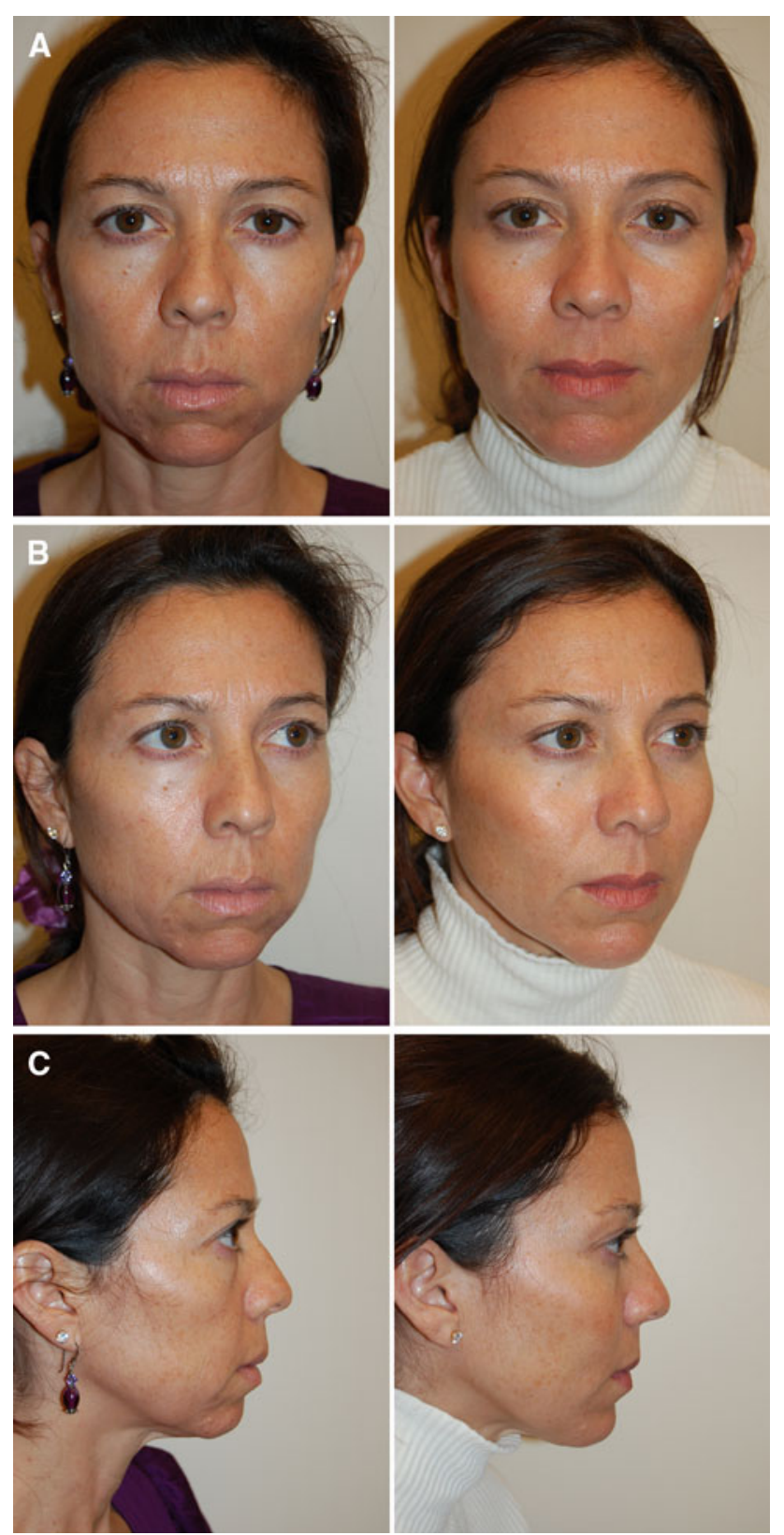

Fig. 6 Frontal (a), oblique (b), and c profile views of 6-month follow-up results for a 37-year-old woman after endoscopic midface lift using lower eyelid fat transposition, endoscopic brow-lift, and augmentation genioplasty with an extended anatomic silicone implant bilateral lower eyelid fat repositioning was less than 20 min.

During this study, one complication developed for a single patient who experienced a temporary dense right lower zygomatic nerve motor palsy with upper lip depression and asymmetry, mild right upper zygomatic branch motor nerve palsy with slightly sluggish eyelid closure, and moderate temporary right frontal branch motor nerve palsy with brow depression (Fig. 7). Contralateral Botox Cosmetic (Allergan, Irvine, CA, USA) injections to the left forehead $(20 \mathrm{u})$ and upper lip (20 units) were performed 3 weeks postprocedurally to improve symmetry, and the patient was started on a facial nerve injury physical therapy regimen. The therapy regimen entailed passive range-of-motion exercises and massage during the acute phase followed by mirror biofeedback for the performance of isolated and precise facial motion during the active phase of recovery. Within 8 weeks, all regions of motor nerve weakness to the right face had completely resolved (Fig. 8a-c).

No other complications in this study, specifically no evidence of lower eyelid malposition, ectropion, entropion, or incomplete release of lower eyelid fat, were encountered. Moderate to significant improvements in tear trough appearance, lower eyelid herniation, and midface positioning were noted in all six patients.

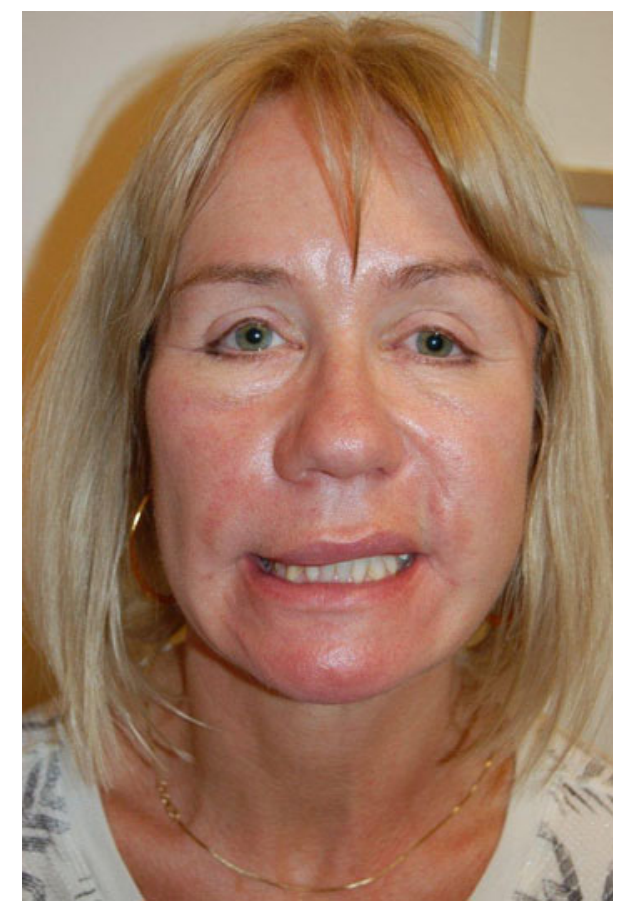

Fig. 7 Results at the 3-week follow-up assessment demonstrating a lower zygomatic branch facial nerve injury in a 49 -year-old woman after endoscopic midface lift with lower eyelid fat transposition, "pinch" lower eyelid blepharoplasty, endoscopic brow-lift, and structural fat grafting to the nasolabial folds 

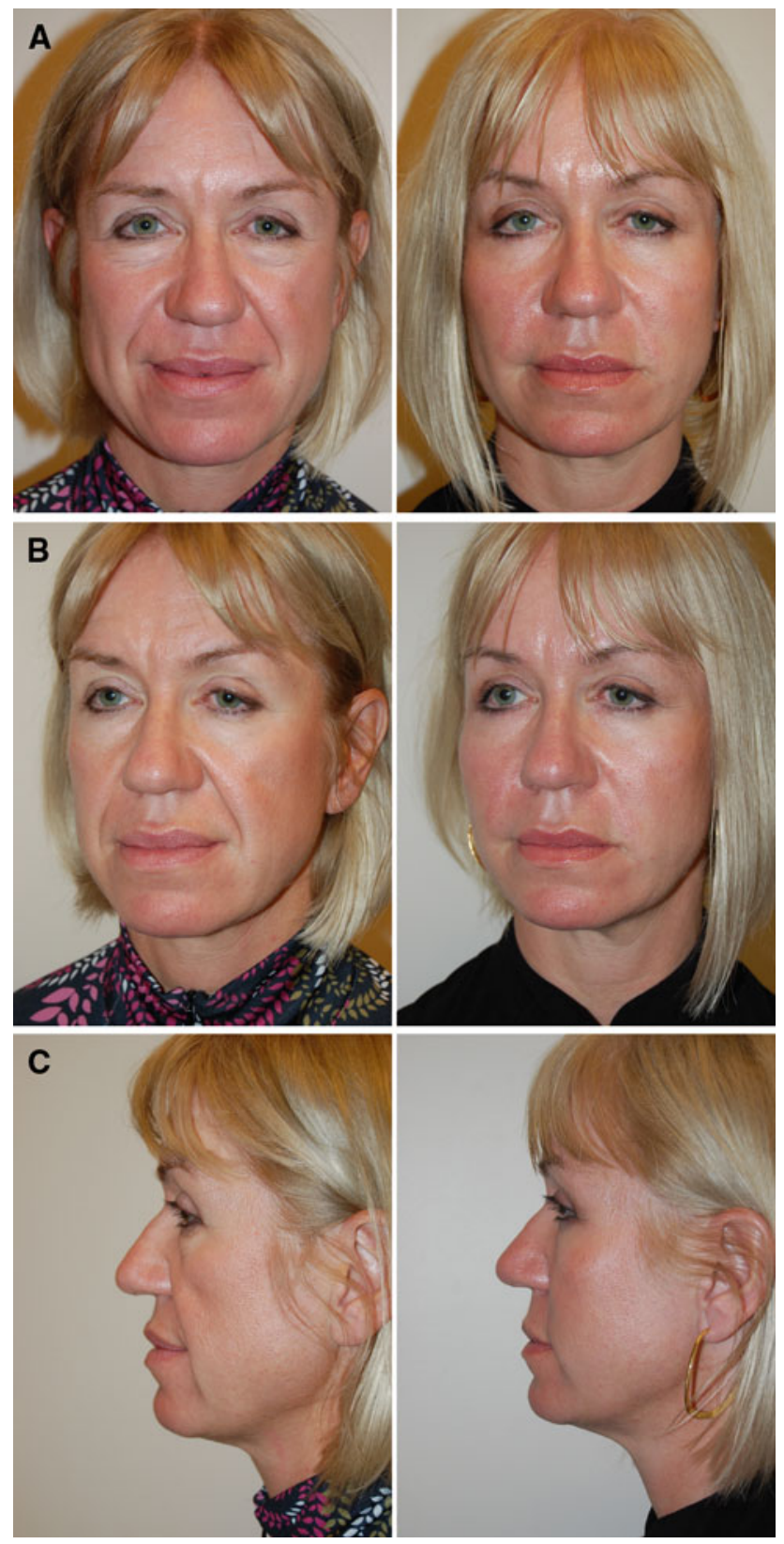

Fig. 8 Frontal (a), oblique (b), and profile (c) views of the patient in Fig. 7 at the 3-month follow-up assessment

\section{Discussion}

For more than a decade, fat preservation techniques to treat lower eyelid surgery have been advocated to avoid complications related to overaggressive fat resection that results in a hollowed orbital appearance, enophthalmos, and worsening of facial aging. For many patients, the problem of lower eyelid fat herniation typically is associated with a deep tear trough and in many cases with midface descent. A technique that combines a raising of the valley and lowering of the hill is an ideal solution to a difficult problem.

Treatments involving the use of injected fat to even the appearance of the eyelid-cheek junction are limited by variable absorption of the fat, asymmetry, unevenness, and coalescence of the fat into palpable fibrotic nodules that are very difficult to treat. The use of nonsurgical fillers to augment the midface and fill the tear trough are temporary and do not treat the underlying problem of associated lower eyelid fat herniation.

To date, transconjunctival and skin-muscle flap approaches have been proposed for lower eyelid fat transposition and midface elevation. However, the limitations of these techniques are obvious. The transconjunctival approach is limited by poor visualization, incapacity to secure midface soft tissues to a stable landmark, postoperative chemosis, very difficult efforts to transpose and secure lower eyelid fat of the lateral fat compartment, and ocular motility restriction due to intraconal scarring [6]. Skin-muscle flap approaches for midface elevation and lower eyelid fat transposition are limited by high rates of lower lid retraction, ectropion, inadequate release of the midface, lateral canthal malpositioning, and thickening of lateral canthal scars used for the enhanced exposure to the midface [7].

Intraoral access to the midface provides enhanced visualization to the orbital septum by the capacity it affords for a large incision and an approach to the septum along its full length from the medial to the lateral fat compartments. Additionally, the exposure allows for far greater midface dissection and elevation with secure fixation to the deep temporal fascia. The additional exposure and release afforded by this technique allows for lower eyelid fat transposition without fixation, unlike the transconjunctival technique, which is limited by poor exposure and inadequate release, prompting the need for suture fixation. The one noted complication of temporary yet dense motor nerve paralysis is likely related to midface dissection and elevation over the zygomatic arch or adjacent nerve traction injury from the midface fixation device used. Temporary zygomatic and frontal nerve injuries in association with the intraoral midface lift and brow-lifting procedures are well documented in the literature and do represent a known risk of the procedure [8].

\section{Conclusion}

A novel approach for lower eyelid fat transposition in combination with midface lift using the intraoral approach is presented. Excellent visualization, decreased operative times, technical ease, and improved outcomes all are potential advantages of this technique in which access to 
lower eyelid fat and midface lift are performed using the transconjunctival or skin-muscle flap approaches. The transoral dissection plane of the midface and over the zygomatic arch is familiar to most plastic surgeons for the treatment of facial fractures. The procedure is easily combined within the same tissue plane using the endoscopic brow-lift procedure. Additionally, lower eyelid skin excess may be treated concurrently using a pinch blepharoplasty technique. Additional study of the midface lift procedure, the long term results, and documentation of the risk for nerve injuries in association with suture and other fixation devices is encouraged.

Acknowledgments The costs associated with the cadaveric dissection in this study were funded by Coapt Systems, Inc. (Palo Alto, CA, USA).

\section{Conflicts of interest None.}

Open Access This article is distributed under the terms of the Creative Commons Attribution Noncommercial License which permits any noncommercial use, distribution, and reproduction in any medium, provided the original author(s) and source are credited.
2. de la Plaza R, Arroyo JM (1988) A new technique for the treatment of palpebral bags. Plast Reconstr Surg 81:677

3. Hamra ST (2003) The role of the septal reset in creating a youthful eyelid-cheek complex in facial rejuvenation. Plast Reconstr Surg 113:2124

4. Patipa M (2004) Transblepharoplasty lower eyelid and midface rejuvenation: Part 1. Avoiding complications by utilizing lessons learned from the treatment of complications. Plast Reconstr Surg 113:1459

5. Goldberg RA (2000) Transconjunctival orbital fat repositioning: transposition of orbital fat pedicles into a subperiosteal pocket. Plast Reconstr Surg 105:749

6. Goldberg RA, Yuen VH (2002) Restricted ocular movements following lower eyelid fat repositioning. Plast Reconstr Surg 110:302

7. Patipa M (2004) Transblepharoplasty lower eyelid and midface rejuvenation: part 2. Functional applications of midface elevation. Plast Reconstr Surg 113:1469

8. De Cordier BC, de la Torre JI, Al-Hakeem MS, Rosenbert LZ, Costa-Ferriera A, Gardner PM, Fix RJ, Vasconez LO (2002) Rejuvenation of the midface by elevating the malar fat pad: review of the technique, cases, and complications. Plast Reconstr Surg 110:1526

\section{References}

1. Camirand A, Doucet J, Haris J (1997) Anatomy, pathophysiology, and prevention of senile enophthalmia and associated herniated lower eyelid fat pads. Plast Reconstr Surg 100:1535 